\title{
A DECENTRALIZED SELF-ORGANIZED APPROACH FOR WIRELESS SENSOR NETWORKS
}

\author{
Jean-Paul Jamont, Michel Occello, André Lagrèze \\ Laboratoire de Conception et d'Intégration des Systèmes \\ Institut National Polytechnique de Grenoble \\ F-26000 Valence, France \\ jean-paul.jamont@esisar.inpg.fr, michel.occello@iut-valence.fr, andre.lagreze@iut-valence.fr
}

\begin{abstract}
This paper deals with an application of multiagent systems to management in wireless sensor networks (WSN). This WSN will be applied to monitor an underground hydrographic network (the EnvSys project). We present the EnvSys project, the multiagent systems (MAS) and their application in WSN. After we propose an adaptive infrastructure of autonomous agents to route the information in the best way, in consideration to strong constraints on energy resources. Interesting simulation results are discussed.
\end{abstract}

Keywords: Multiagent system, wireless sensor network, self-organization.

\section{INTRODUCTION}

Considering complex embedded control systems as decentralized cooperative nodes networks is a recent but attractive way to design intelligent applications.

In some cases, especially for aggressive environment applications, nodes cannot be interconnected through classical field buses and wireless technology is required. The whole system becomes an open network of intelligent autonomous embedded entities controlling sensors and actuators.

We introduce in this paper a multiagent approach to design such applications. Further, we intend to show that using behavior modelling, cooperative aspects and organizational techniques of multiagent systems allows increasing the overall efficiency of embedded systems.

In a first section we describe the EnvSys project, an underground river instrumentation system, which motivates this study. We expose in a sec- 
ond section multiagent systems (MAS) and their application to wireless intelligent sensor networks (WSN). We then propose our multiagent approach based on self-organization to manage the functional integrity of the decentralized embedded nodes network. Finally, after a presentation of our simulation platform and of some quantitative results, we give an insight to the operational embedded architecture.

\section{THE ENVSYS PROJECT}

\subsection{Origin and issue}

The purpose of the ENVironment SYStem project is to monitor an underground river system (Jamont, J.-P. et al, 2002). At the origine of this project are the difficulties to measure various parameters in an underground river system is a complex task : access to this type of underground galleries requires help from speleologists, installation of wire communications networks is difficult, especially because an hydrographic system has a chaotic structure and, in the case of radio communication, the underground aspect complicate wave propagation (these techniques are not totally mastered).

The general idea of the project is to study the feasibility of a WSN from an existing physical layer. This will allow wireless instrumentation of a subterranean river system. Such a network would present an important interest in many domains: the study of underground flows, the monitoring of deep collecting, flooding risk management, river system detection of pollution risks, etc.

In a subterranean river system, the interesting parameters to measure are numerous: temperature of air and water, air pressure and if possible water pressure for the flooded galleries, pollution rate by classical pollutants, water flow, draft speed, etc. All this information will be collected at the immediate hydrographic network exit by a work station like a PC. These data will be processed to activate alarms, study the progress of a certain pollution according to miscellaneous measuring parameters, determine a predictive model of the whole network by relating the subterranean parameters measures of our system with the overground parameters measures more classically on the catchment basin.

Every sensor has a limited transmission range due to rock blocks properties. This limitation results from three points: the technological solutions which are used to achieve the sensor transmission module (frequency, power, antenna), the implementation of these solutions and, finally, the environment. Indeed, according to the obstacles it will have to go through, the electromagnetic waves will not be usable at the same 
distance for each direction. The transmission zone will not be modeled by a sphere.

\subsection{Difficulties of communication management in wireless sensor networks}

WSN are a particular type of adhoc network. In adhoc network, communication between two hosts is generally not direct. To communicate, they require help from others host. It is a multihop communication. Thus, the sensors have two tasks : make their own measure and assure routing functionnality for others sensors. This multihop caracteristic create an important routing problem (routing consist to find the path between a sender to a receiver node) because all the adhoc routing protocol use flooding technics (in a flooding scheme a host give the message to all its neigboors etc.) and the location updating is difficult (location updating consist to maintening information about its neighboors).

A lot of problems are added in the case of WSN. An overview of these difficulties, collectively accepted today, is given in (Zhang, W. et al, 2002). Some of them concern the sensors (hardware limitation for financial cost reason) and its energy ressource. Sensor's battery are difficult to replace, one of the global system aim so to reduce as possible the energy cost. When it has nothing to do generally for conserving energy they enter in a sleep mode. When they communicate they must use good routing protocol and optimal way (generally the criteria will be the number of hop). An agressive environment like the underground river system can cause some internal fault for sensors. So WSN must be very adaptive, fault tolerant and self-stabilized : a sensor failure must not have an important impact on the system. This system must provide reliable communication and, sometimes, adapt to "real-time" constraints. Furthermore, in the case of mobile devices the infrastructure of sytems are not persistant.

Our work deals with the analysis of the problem using a MAS approach. The main contribution of the work presented in this paper is situated at a logical level : we don't discuss about the physical layer. We talk about the monitoring of this system, the complex environment, the kind of intelligence giving to the network.

\section{MULTIAGENT AND WSN}

\subsection{Agents and multiagents systems}

An agent is a software entity endowed with autonomous behaviors and embedded in an environment which it can perceive and in which it acts. 
This entity has its own objective. Autonomy is the main concepts in the agent issue: it is the ability of agents to control their actions and their internal states. The autonomy of agents implies no centralized control. The power of an agent decomposition is the decentralization of the intelligence, i.e. the decision capabilities, and of entities' knowledge.

A MAS is a set of agents situated in a common environment, which interact and attempt to reach a set of goals. Through these interactions a global behavior can emerge. The emergence process is a way to obtain, from cooperation, dynamic results that cannot be predicted in a deterministic way.

The multiagent methods aim at decreasing the complexity of system design by a decentralized analysis. There are several MAS methods ( Iglesias, C. et al, 1998) among which most are centered on the analysis of agents'tasks as the methods Gaia and MaSE, others on the roles or on the organization as the method AALAADIN.

We are thereafter going to be interested in the AEIO decomposition. We will follow the method of multiagent design discussed in (Occello and Koning, 2000), associated to this MAS decomposition. It proposes a decomposition according to four axes collectively accepted today. The agent axis (A) gathers all elements for defining and constructing these entities. The environment axis (E) deals with elements necessary for the MAS realization such as the perception of this environment and the actions one can do on it. The interaction axis (I) includes all elements which are in use for structuring the external interactions among the agents (agent communication language, interaction protocols) The organization axis $(\mathrm{O})$ allows to order agent groups in organization determined according to their roles.

We chose to apply this multiagent method for our problem because it privileges an explicit description of the interactions and the environment.

\subsection{Wireless sensor networks management}

WSN management and MAS. The distributed and open nature of WSN means that the MAS approach is an adapted answer. Another advantage of this approach is the external representation of the interactions and of the organization. External representations offer multiple possibilities such as the monitoring by an external observer.

A few works reaching the same objectives show that the approach is interesting. We can quote the ActComm (Gray, 2000) project which is a military project for which the routing of information is essential: it aims at studying the communication management between a soldier team and a military camp via a satellite. We can also mention the work of (Petriu, 
E.M. et al, 2002) on wireless networks of mobile autonomous intelligent sensors where agents are used to achieve flexible and open cell assembly. Another example is the Unmanned Ground Vehicle Program ARPA's project (Cook,D.J. et al, 1996) which approaches the information management resulting from a group of autonomous observation military vehicles. The problem described in (Zhang, W. et al, 2002) is very similar to our problem but the approach is very different since the used technique is based on distributed stochastic algorithms.

MAS are used in very active way for service descriptions and service discovery in ad-hoc networks (Chen, H. et al, 2000).

WSN and routing protocol. The WSN associated routing protocols are centered on the flooding techniques. There are three differents type of routing protocols families. The reactive protocols using no routing table. The main idea of this family is to reduce the flows by creating clusters for example. The proactive protocols using routing tables, periodically updated, and for those it is necessary to exchange control packets (energy cost). The hybrid protocols adopting the reactive protocol behavior and, if necessary, using routing tables for increasing efficiency.

\section{A MAS FOR WSN COMMUNICATION MANAGEMENT}

\subsection{AEIO analysis of this problem}

As previously examined, this approach is articulated around four axes.

The environment axis. The environment will be made of the measurable information. It is deterministic, non episodic, dynamic and continuous. Agents are situated but don't know their position.

The organization axis. In this type of application no one can control the organization a priori. Relations between agents are going to emerge from the evolution of the agents'states and from their interactions. We are going to be content with fixing the organization parameters, i.e. agents'tasks, agents'roles.

The organizational basic structures (see fig 1) are composed of one group representative agent (managing the communication in his group), some connection agents (they know different representative agents and they can belong to several groups) and some ordinary members (active in the communication process only for their own tasks. They don't ensure information relay). 


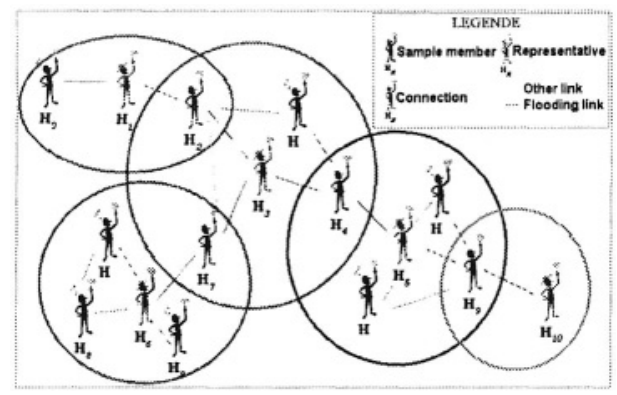

Figure 1. Group organisation for communication management

Because a representative agent is the most sollicited agent in a group, the best one having the most important level of energy and the most important number of neighbors. We use a role allocation based selforganization mechanism involving the election of a representative agent based on a function which estimates the adequation between it desire to be the boss and its capacity to be. The organization is modified only when a problem occurs. We don't try to maintain it if we have no communication.

The interaction axis. The agents will interact only with the agents in acquaintance (an agent is in acquaintance with another if it is aware of its existence). Agents interact by asynchronous exchange of messages (without rendez vous). Among the different protocols that we use, the choice of an introduction protocol is essential. Indeed, this protocol allows to the agents to be known, i.e. to bring their knowledge and their know-how to the agents' society. We defined thirteen different types of small messages.

The agent axis. Sensors are modeled by agents. These agents have hybrid architectures, i.e. a composition of some pure types of architectures. Indeed, the agents will be of a cognitive type in case of a configuration alteration, it will be necessary for them to communicate and to manipulate their knowledge in order to have an efficient collaboration. On the other hand, in normal use it will be necessary for them to be reactive (stimuli/response paradigm) to be most efficient.

The agents have then to achieve a measuring function (the main work of a sensor, it consists in interacting with the environment to acquire information about one of the environment parameters) and a communication task for giving (if necessary) the information to other devices or relay- 
ing neighbor's messages. All the agents have the same communication capabilities but the communicated data depend of their roles.

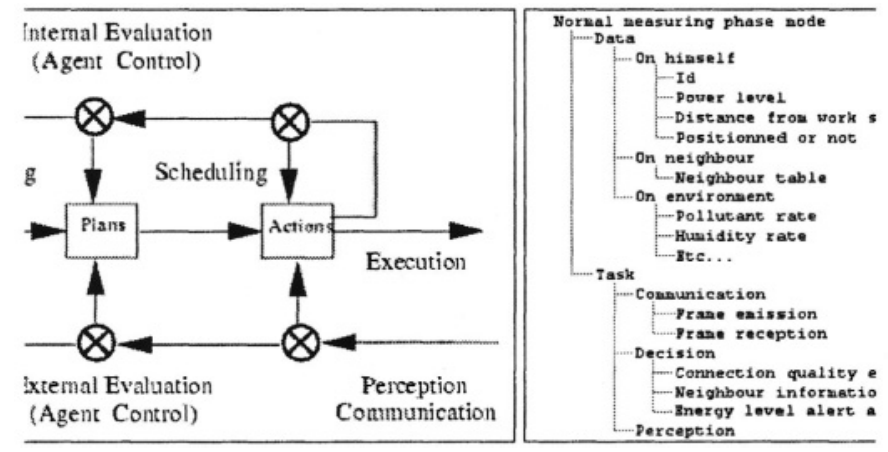

Figure 2. Agent architecture and agent tasks tree

Using a hybrid architecture for the agents enables to combine the strong features of each of reactive and cognitive capabilities seen before. The ASTRO hybrid architecture (Occello, M. et al, 1998) is especially adapted to a real time context. The integration of deliberative and reactive capabilities is possible through the use of parallelism in the structure of the agent. Separating Reasoning/Adaptation and Perception/Communication tasks allows a continuous supervision of the evolution of the environment. The reasoning model of this agent is based on the Perception/Decision/Reasoning/Action paradigm. The cognitive reasoning is thus preserved, and predicted events contribute to the normal progress of the reasoning process.

\section{THE EXPERIMENTATION}

In order to evaluate and improve such agents' software architectures and the cooperation techniques that they involve, we introduce a simulation stage in our development process.

\subsection{Simulation Results}

The simulation first allowed us to experiment our approach and the software solutions that we provide for the various problems. We can also quantify the emergence inferred by the MAS approach in this case.

The simulation software structure is very basic. In fact, we have two types of components: SimSensor and SimNetwork. A SimSensor component simulates the sensor behavior. It possesses its own model and architecture. All the sensors have the same communication capabilities. 
They transmit their requests to the SimNetwork component sends this information to all sensors which can receive them, in the environment. SimNetwork can appear as the inference mechanism for the simulation.

We have compared our MAS to three traditionnal ad-hoc protocols. The DSDV protocol and the natural DSR protocol do not appear in this comparaison because its efficiency were lower than the ehanced version of DSR which use a route maintenance (memorization of main route).

Use case 1. At a first, let us present some performances in the ENVSYS context. All sensors communicate only with the workstation situated at the end of the undergound river system : it is a unidirectionnal protocol. In this case, messages are small (one byte for data type and four for the measure). For this example, three messages are send by five second. The same scenario is applied for the different protocols.
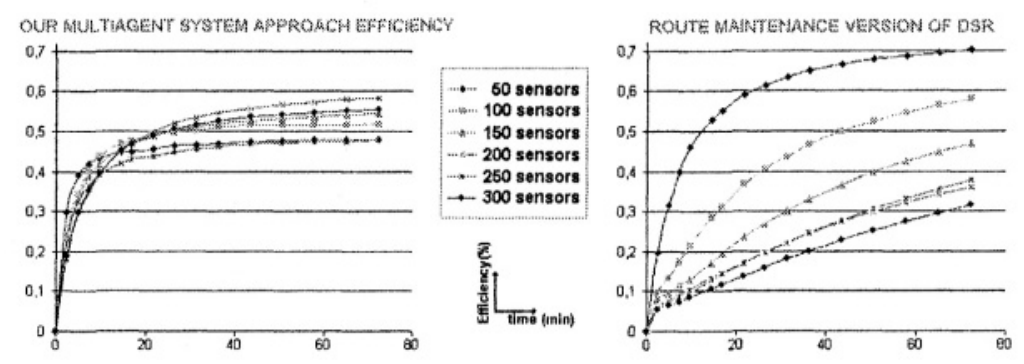

Figure 3. Approach comparison for unidirectionnal use case

We can see that the benefit (fig 3) of our approach is important. Quickly our routing method can deliver all messages with a good efficiency. Higher is the number of sensors better is the reactivity of our approach. We must note that if the system knows no pertubation or mobility variation of DSR will be better from an efficiency point of view. It is normal because in this case DSR learns all the routes (succession of sensors) allowing to communicate with the workstation. It is not really the case of our approach witch reason from the group and not from the sensors. One consequence is that the route used by the messages with our approach are not optimal.

Use case 2. In this case, we are in the ENVSYS context where the sensors communicate together for elaborating more complex measures. We choose to give to the message a size of thirty bytes. In this case the behavior of our approach is much better than DSR because is route management it more complicated. If we add some perturbations on these 

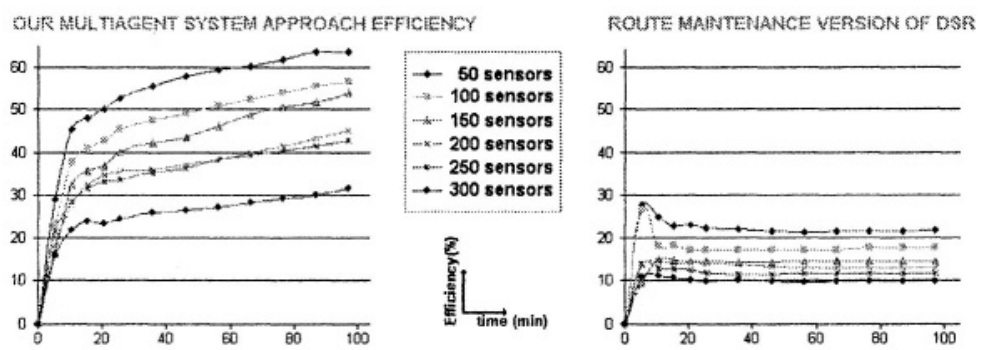

Figure 4. Approach comparison for the multidirectionnal use case

scenarios (one perturbation by three minutes) the efficiency is nearly the same (it is not the case for the DSDV protocol).

\subsection{The operational embedded architecture}

Therefore, we will demonstrate the feasibility of our approach. For the sensors we have chosen a classical three-layers architecture (physical layer/Link layer/Applicative layer).

We use the physical layer which is employed by NICOLA system, a voice transmission system used by the French speleological rescue teams (Graham, 1999). This layer is implemented in a digital signal processor rather than a full analogic system. Thereby we can keep good flexibility and we are able to apply further a signal processing algorithm to improve the data transmission.

The link layer used is a CAN (Controller Area Network) protocol stemming from the motorcar industry and chosen for its good reliability. The applicative layer is constituted by the agents' system. The agents are embedded on autonomous processor cards. These cards are equipped with communication modules and with measuring modules to carry out agent tasks relative to the instrumentation. These cards supply a real time kernel. The KR-51(the kernel's name) allows multi-task software engineering for $\mathrm{C} 515 \mathrm{C}$ microcontroller. We can produce one task for one capability. We can then quite easily implement the parallelism inherent to agents and satisfy the real-time constraints.

\section{CONCLUSION}

This software agent architecture is embedded on autonomous processor cards. The MAS, which we are creating, is open: adding a sensor does not require a manual reconfiguration. Most of sensors'dysfunctions should not threaten the functional integrity of the whole system which 
should be fault tolerant. All the sensors have a hybrid decisionnal architecture based on the ASTRO model. Through the simulation step, we can already notice what the MAS approach provides:

1. The emergent feature, which is inferred by the MAS, makes the system fault tolerant to changes of the environment in which it evolves.

2. Agents present interesting features of software engineering such as genericity allowing an easy evolution of the applications.

3. Generic aspects of agents allows us to envisage differents applications for this network type such as diagnosis, risk management, data fusion...

In a near future, we want to analyse the effect of a recursive mechanism on this application to increase its efficiency in the case of a very pertubated context. We project to apply our approach to other applications such as health monitoring and movement tracking. For these applications, eavesdrop can introduce new problems on messages security.

\section{REFERENCES}

Chen, H. et al (2000). Service discovery in the future electronic market. In Working notes of Seventeenth National Conference on Articial Intelligence, Eleventh Innovative Applications of AI Conference, Austin.

Cook,D.J. et al (1996). Decision-theoric cooperative sensor planning. IEEE Transactions on Pattern Analysis And Machine Intelligence, 18.

Graham, N. (1999). The Nicola Mark II a New Rescue Radio for France. In The CREG Journal, volume 38, pages 3-6.

Gray, R. (2000). Soldiers, agents and wireless networks: A report on a military application. In Proceedings of the Fifth International Conference and Exhibition on the Practical Application of Intelligent Agents and Multi-Agents, Manchester, England.

Iglesias, C. et al (1998). A survey of agent oriented methodologies. In Proceedings of ATAL 98 - Workshop on Agent Theories, Architectures, and Languages, volume LNAI 1555, pages 163-176, Paris, France. Springer-Verlag.

Jamont, J.-P. et al (2002). A multiagent system for the instrumentation of an underground hydrographic system. In Proceedings of IEEE International Symposium on Virtual and Intelligent Measurement Systems, Mt Alyeska Resort, AK, USA.

Occello, M. and Koning, J. (2000). Multi-agent based software engineering: an approach based on model and software reuse. In From Agent Theory to Agent Implementation II - EMCSR 2000 Symposium, pages 645-657, Vienna.

Occello, M. et al (1998). Designing organized agents for cooperation in a real time context. In Drogoul, A., Tambe, M., and Singh, J., editors, Collective Robotics, volume LNCS/LNAI 1456, pages 25-73. Springer-Verlag.

Petriu, E.M. et al (2002). Intelligent robotic sensor agents for enviroment monitoring. In Proceedings of IEEE International Symposium on Virtual and Intelligent Measurement Systems, pages 19-20.

Zhang, W. et al (2002). Distributed problem solving in sensor networks. In $A A$ $M A S^{\prime} 02$, pages $15-19$. 